\title{
RESEARCH PAPER \\ REGIONAL CEREBRAL RELAXATION TIMES MEASURED BY MAGNETIC RESONANCE IMAGING AT 3.0 TESLA
}

\author{
A. N. Mumuni ${ }^{1}$, J. McLean ${ }^{2}$ and B. Condon ${ }^{2}$ \\ ${ }^{1}$ Department of Human Biology, School of Medicine and Health Sciences, \\ University for Development Studies, Tamale, Ghana \\ ${ }^{2}$ MRI/SPECT Unit, Institute of Neurological Sciences, Southern General Hospital, \\ 1345 Govan Road, Glasgow G51 4TF, Scotland, UK \\ Corresponding author: Email: mnashiru@uds.edu.gh
}

\begin{abstract}
Brain tissue $T_{1}$ and $T_{2}$ relaxation times were measured at $3.0 \mathrm{~T}$ from a total of $8(M / F=5 / 3)$ healthy volunteers, selecting 9 regions of interest (ROIs) in the prefrontal, frontal, parietal, occipitoparietal and hippocampal regions. Apart from the prefrontal region, the other four ROIs were selected within the left and right brain hemispheres. The selected ROIs contained either grey matter (GM) or white matter (WM) or both. The $T_{1}$ measurements were done using the saturation recovery imaging method at 8 different repetition times (TRs) whereas the $T_{2}$ measurements were carried out using the multiple spin-echo imaging method at 12 different echo times (TEs). The average $T_{1}$ values (mean $\left.\pm S E, m s\right)$ from $4(M / F=2 / 2)$ volunteers were: $1942 \pm$ 29 (prefrontal GM), $1203 \pm 40$ (frontal WM), $1217 \pm 21$ (parietal WM), $1425 \pm 29$ (occipitoparietal GM/WM), and $1435 \pm 62$ (hippocampi). The average $T_{2}$ estimates (mean $\pm S E$, ms) from another group of $4(M / F=3 / 1)$ volunteers were: $147 \pm 9$ (prefrontal GM), $121 \pm 3$ (frontal WM), $131 \pm 4$ (parietal WM), $127 \pm 1$ (occipitoparietal GM/WM), and $142 \pm 8$ (hippocampi). Neither $T_{1}$ nor $T_{2}$ relaxation times differed significantly between the two brain hemispheres by paired t-tests $(p>0.05)$. However, regional $T_{1}$ was found to vary significantly ( $p$ $<0.01)$ while regional $T_{2}$ did not vary significantly $(p=0.07)$ by one-way ANOVA. These findings are consistent with theory and published data for the ROIs studied. The results could thus serve as a reference data set for brain MRI pulse sequence optimisation at $3.0 \mathrm{~T}$ and could as well be useful in multicentre data set comparisons aimed at developing a database of in vivo brain relaxation times.
\end{abstract}

Keywords: MRI, brain, saturation recovery, spin echo, relaxation time

\section{INTRODUCTION}

The choice of a suitable pulse sequence to achieve a desired image contrast in magnetic resonance imaging (MRI), and the optimisation of any MRI method at 3.0 $\mathrm{T}$ or a greater field strength depend on the knowledge of the fundamental nuclear magnetic resonance (NMR) properties of biological tissues at the given field strength (Wansapura et al., 1999; Kim et al., 1994). Accurately quantified tissue NMR 
relaxation times are important for characterising these tissue NMR properties and for optimising contrast in relatively new imaging methods such as functional MRI, spectroscopic imaging and perfusion imaging (Wansapura et al., 1999). The two most important relaxation times that are usually measured in vivo in this regard are the spin-lattice $\left(T_{1}\right)$ and spin-spin $\left(T_{2}\right)$ relaxation times.

$T_{1}$ relaxation time strongly depends on the external magnetic field strength, $B_{\mathrm{o}}$ (Koenig and Brown, 1984). Even though $T_{1}$ may be estimated at any field strength based on theoretical predictions, it is suggested that such derived results will be significantly erroneous and may not be adequate for accurate contrast calculations (Wansapura et al., 1999). Brain tissue $T_{2}$ relaxation time on the other hand partly depends on $B_{0}$ but there is no evidence for this behaviour from theory (Wansapura et al., 1999; Posse et al., 1995). Consequently, quantitative measurements of brain tissue NMR relaxation times at $B_{\mathrm{o}} \geq 3.0 \mathrm{~T}$ are needed and will serve as essential reference data for advanced MR imaging and other quantitative NMR techniques such as MR spectroscopy. Furthermore, reference relaxation data in healthy brain is essential for their diagnostic value as they vary in disease (Laule et al., 2007; Whittall et al., 1999).

However, it appears from the literature that investigations of relaxation times of human brain tissue at field strengths of $3.0 \mathrm{~T}$ and above are limited. Secondly, due to the differences in the chosen regions of interest (ROIs), some brain regions, including those investigated in this report, have been considered by very few research groups. The objective of this study therefore was to accurately measure relaxation times at $3.0 \mathrm{~T}$ of both grey matter (GM) and white matter (WM) structures in normal human brain, using saturation recovery and multiple spin-echo imaging methods for $T_{1}$ and $T_{2}$ measurements, respectively. The measured relaxation times could be applicable in future studies such as those aimed at MRI pulse sequence optimisation and diagnostic MRI (where pathology is associated with changes in relaxation times).

\section{MATERIALS AND METHODS \\ Subjects}

With permission from the West of Scotland Research Ethics Committee 4 (WoSREC4), a total of 8 ( 5 males, 3 females) healthy subjects participated in the study. Brain tissue $T_{1}(\mathrm{M} / \mathrm{F}=$ $2 / 2$, mean age $=32.0$ years $)$ and $T_{2}(\mathrm{M} / \mathrm{F}=3 / 1$, mean age $=32.5$ years $)$ relaxation times were each measured separately in 4 subjects. The 4 subjects in each one of the two sets of measurements were randomly assigned. Each volunteer gave prior informed written consent. No volunteer had any neurological or psychiatric disorder.

\section{Magnetic resonance imaging}

Magnetic resonance imaging for the measurement of both relaxation times were performed on a 3.0 T GE MR scanner equipped with an eight-channel receive-only head coil.

For the measurements of both $T_{1}$ and $T_{2}$, axial slices covering the whole brain were planned along the hippocampal angle. Each slice was 3 $\mathrm{mm}$ thick without slice gaps; and a matrix of $256 \times 128$ pixels was used to give a reasonable compromise between image resolution and total experimental time. A field of view (FOV) of $25.6 \times 25.6 \mathrm{~cm}^{2}$ was selected to cover the entire axial image, including the hippocampi. For each subject, a high resolution $T_{1}$-weighted $3 \mathrm{D}$ image of the whole brain was also acquired using an axial slab along the hippocampus. This high resolution axial $3 \mathrm{D}$ image gave a better grey/white matter contrast than the corresponding $T_{1}$ - and $T_{2}$-weighted images. These highresolution $3 \mathrm{D}$ MR images served as references during ROI selection in the respective $T_{1^{-}}$and $T_{2}$-weighted images by co-registration of a given relaxation time weighted image to its corresponding 3D image. Thus all images of any one volunteer acquired within the same scan session for the estimation of either $T_{1}$ or $T_{2}$ were corrected for rotational errors. The advantage of using axial slices for this study is that in 
all acquisitions, they provide access to slices that contain sufficient grey and white matter for the placement of ROIs. Measurements of the $T_{1}$ and $T_{2}$ relaxation times were performed in separate examination sessions as follows.

\section{$T_{1}$ Measurements}

$T_{1}$ measurements were carried out using a saturation recovery method with a variable $T R$ spinecho imaging sequence. A constant echo time (TE) of $11.0 \mathrm{~ms}$ was maintained, while axial images (Fig. 1) were acquired at eight successive $T R$ times of 200, 350, 550, 750, 1000,
1500, 2000 and $4000 \mathrm{~ms}$

\section{$T_{2}$ Measurements}

For $T_{2}$ measurements, a multi spin-echo pulse sequence was used. A constant $T R$ value of $3000 \mathrm{~ms}$ was maintained and twelve $T_{2}$ weighted images (Fig. 2) were acquired using the following $T E$ values: $16,25,30,32,48,50$, $60,64,75,90,100$ and $120 \mathrm{~ms}$. The multi spinecho pulse sequence on the GE MR scanner used for the measurements produces images at four echo times for each user-selected $T E$ value. The $T E$ values of these acquired images

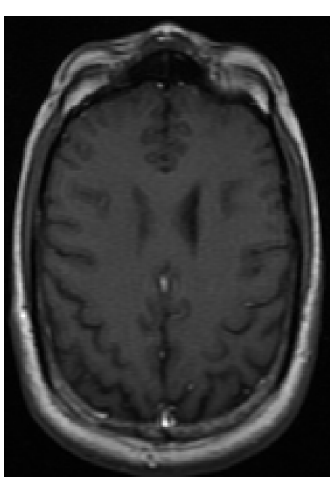

$T R=200 \mathrm{~ms}$

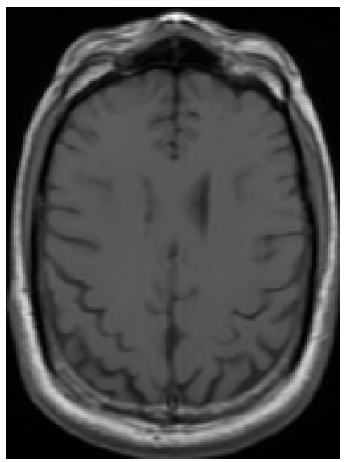

$T R=1000 \mathrm{~ms}$

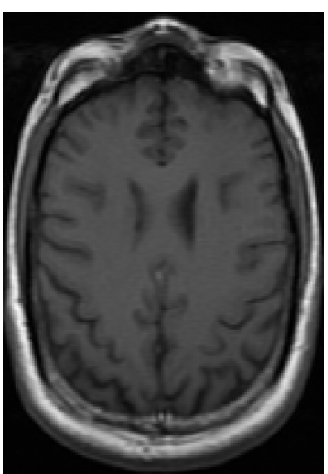

$T R=350 \mathrm{~ms}$

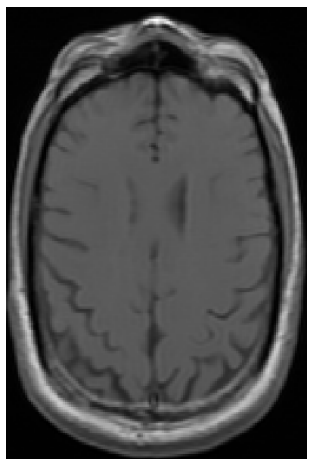

$T R=1500 \mathrm{~ms}$

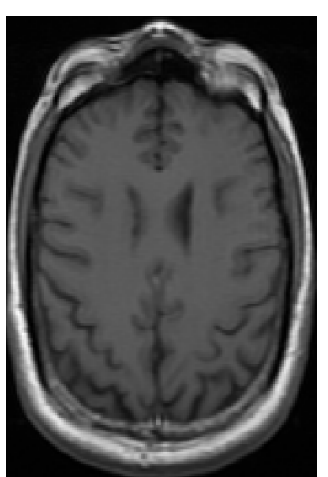

$T R=550 \mathrm{~ms}$

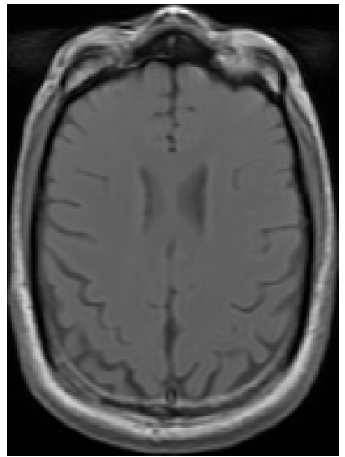

$T R=2000 \mathrm{~ms}$

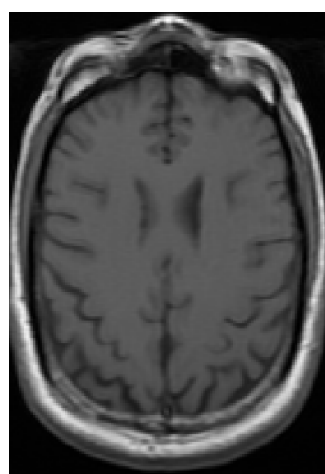

$T R=750 \mathrm{~ms}$

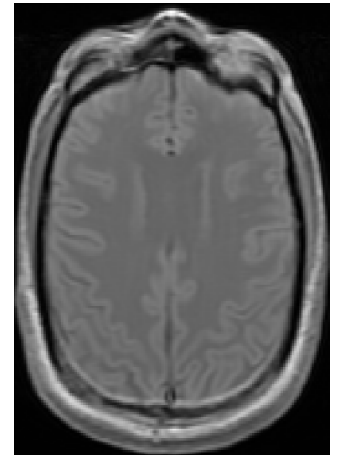

$T R=4000 \mathrm{~ms}$

Fig. 1: Stack of axial MR images of a healthy subject acquired at varying repetition times (TR) using a constant echo time (TE) of $11.0 \mathrm{~ms}$

Note the increasing image intensity as TR increases from $200 \mathrm{~ms}$ (top row) to $4000 \mathrm{~ms}$ (bottom row). These intensities were measured and the values obtained were then plotted against their respective TR values. A nonlinear least square monoexponential recovery (curve) fitting was then performed on the plot to estimate $T_{1}$ relaxation time. 


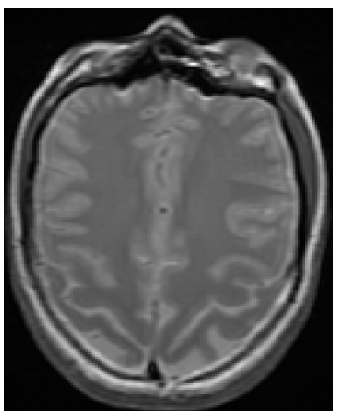

$T E=16 \mathrm{~ms}$

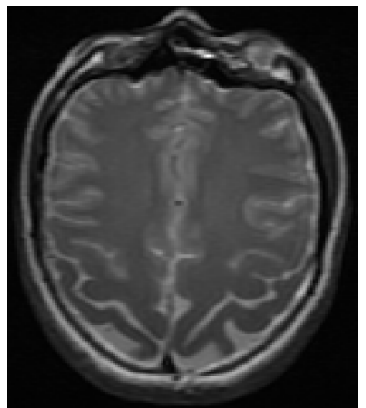

$T E=48 \mathrm{~ms}$

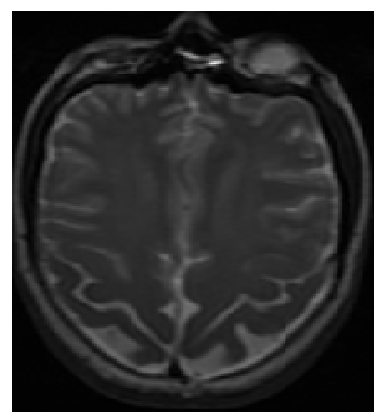

$T E=75 \mathrm{~ms}$

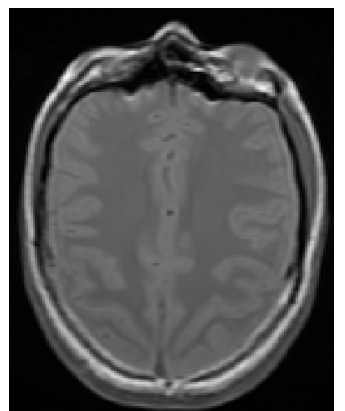

$T E=25 \mathrm{~ms}$

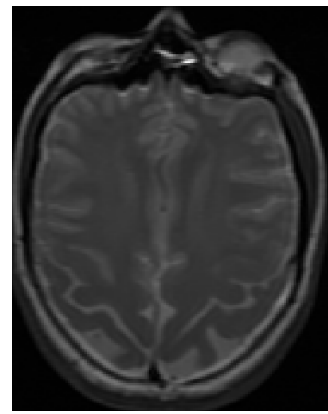

$T E=50 \mathrm{~ms}$

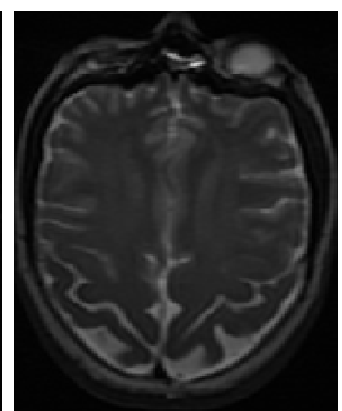

$T E=90 \mathrm{~ms}$

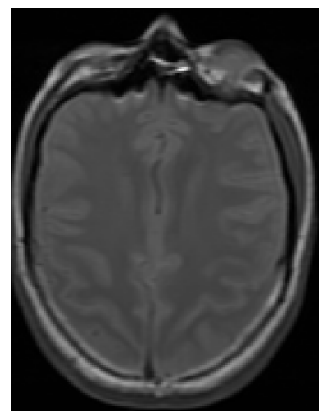

$T E=30 \mathrm{~ms}$

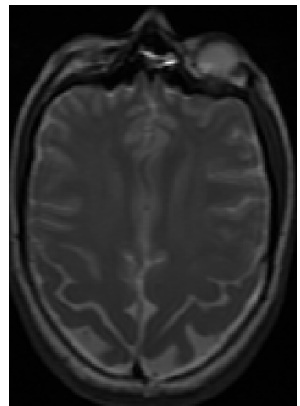

$T E=60 \mathrm{~ms}$

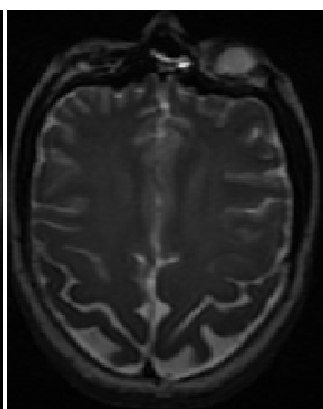

$T E=100 \mathrm{~ms}$

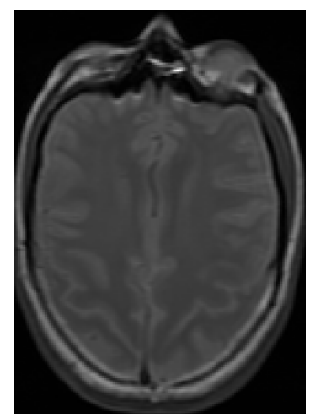

$T E=32 \mathrm{~ms}$

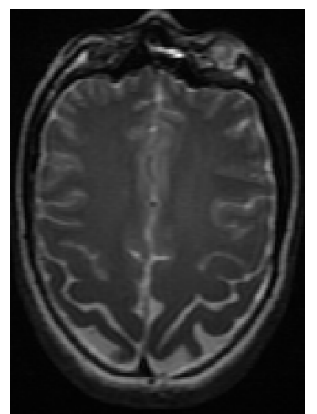

$T E=64 \mathrm{~ms}$

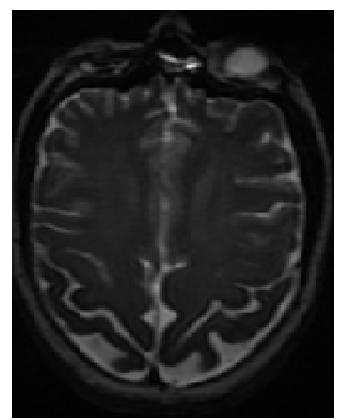

$T E=120 \mathrm{~ms}$

Fig. 2: Stack of axial MR images of a healthy subject acquired at varying echo times (TE) using a constant repetition time (TR) of $3000 \mathrm{~ms}$

Note the decreasing image intensity as TE increases from $16 \mathrm{~ms}$ (top row) through $48 \mathrm{~ms}$ (middle row) to $120 \mathrm{~ms}$ (bottom row). These intensities were measured and the values obtained were then plotted against their respective TE values. A nonlinear least square monoexponential decay (curve) fitting was then performed on the plot to estimate $T_{2}$ relaxation time.

are multiples of the user-selected $T E$ value. For the $T_{2}$ measurements in this study, three $T E$ values were selected: 16, 25 and $30 \mathrm{~ms}$. Thus
$T E=16 \mathrm{~ms}$ produced images at $T E$ times of 16 , 32,48 and $64 \mathrm{~ms}$. The $T E$ values of 25 and 30 $\mathrm{ms}$ also produced four images each, resulting in 
twelve image data sets at the above $T E$ times.

\section{ROI selection and estimation of relaxation times}

Using the $T_{1}$ - and $T_{2}$-weighted images acquired (Figs 1 and 2), four anatomical sites were chosen in each hemisphere, plus one prefrontal region of the brain. This resulted in a total of nine ROIs per subject, as shown in Fig 3a-b. Care was taken in selecting the ROIs, which did not include the ventricles or extra-axial CSF. The shapes of the ROIs were circular, rectangular or freehand-drawn (in the Image-J software, version 1.46r), depending on which shape best-fitted the target area.

Estimates of the brain tissue $T_{1}$ relaxation times were obtained from a nonlinear least square monoexponential recovery fit of the image intensities, $\mathrm{A}_{\mathrm{z}}(T R)$ (measured from each ROI labelled 1-9 in Fig 3a-b) at their respective $T R$ values. The fitting method solved the following equation for $T_{1}$

$\mathrm{A}_{\mathrm{z}}(T R)=\mathrm{A}_{\infty} \mathrm{x}\left[1-\exp \left(-T R / T_{1}\right)\right]$

where $\mathrm{A}_{\infty}$ is the image intensity at the longest possible $T R$ time.

Estimates of the brain tissue $T_{2}$ relaxation times on the other hand were obtained from a nonlinear least square monoexponential decay fit of the image intensities, $\mathrm{A}(T E)$ (measured from each ROI labelled 1-9 in Fig 3a-b) at their corresponding $T E$ times. The fitting method solved the following equation for $T_{2}$ :

$\mathrm{A}(T E)=\mathrm{A}_{\mathrm{o}} \exp \left(-T E / T_{2}\right)$

where $\mathrm{A}_{\mathrm{o}}$ is the maximum image intensity at $T E$ $\approx 0 \mathrm{~ms}$. (a)

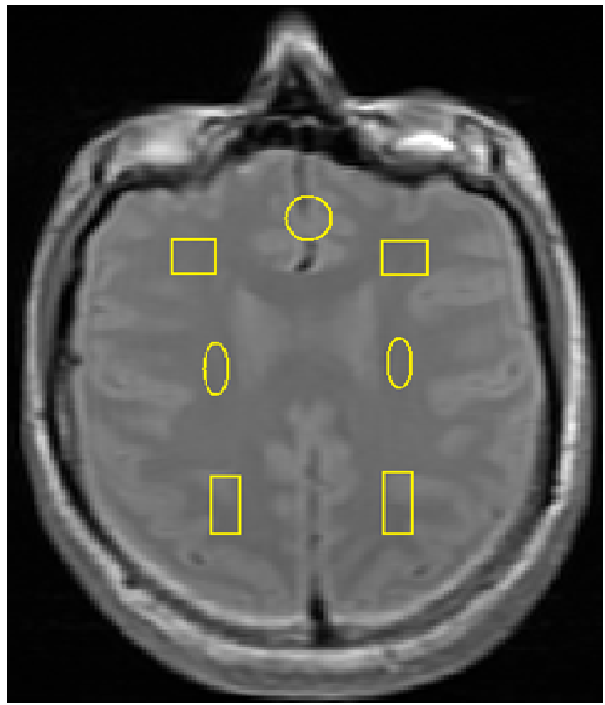

(b)

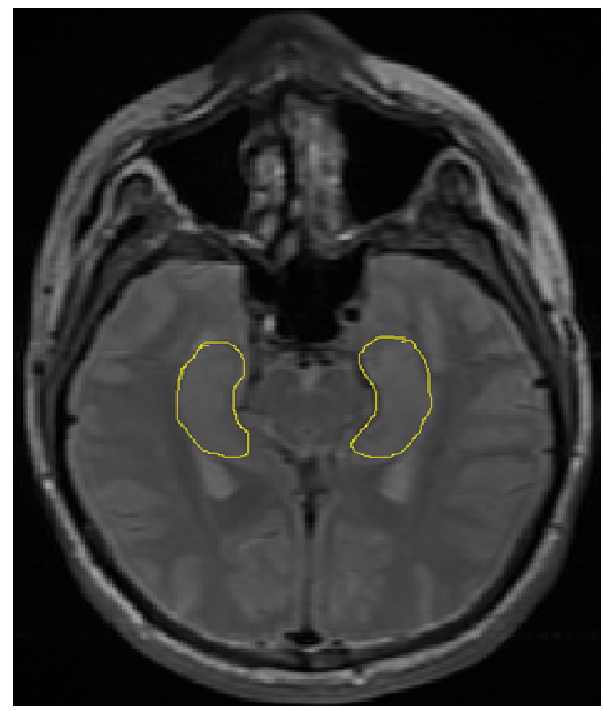

Fig. 3: MRI of an axial slice through the prefrontal brain region (a) and the bilateral hippocampi (b) in a healthy volunteer

The $3 \mathrm{~mm}$ thick slices shown in Fig 3a-b are typical of those chosen for all data analysis. The ROIs are indicated by a circle (1), squares (2 and 3), ovals (4 and 5), rectangles (6 and 7) and freehand-drawn (8 and 9) selections. The numbers correspond to the brain structures listed in Tables 1-3. 


\section{3}

Mumuni et al.

Curve fitting for the estimation of both $T_{1}$ and $T_{2}$ relaxation times were performed using the curve fitting toolbox in MATLAB (version 7.8.0.347, R2009a).

\section{Statistical analysis}

Measured relaxation times from ROIs drawn in the left and right hemispheres were compared using the paired t-test. Assessment of the variations of both relaxation times across the nine ROIs was performed by one-way ANOVA. The two statistical tests were chosen after the data was tested to follow a normal distribution using the Anderson-Darling Normality Test. All statistical tests were performed using the Minitab software package (version 16, Minitab Inc.). A critical value of $p<0.05$ was used to accept a statistically significant difference in the comparisons.

\section{RESULTS}

Averages of the $T_{1}$ and $T_{2}$ relaxation times $(n=$
4 in each case) measured from the nine ROIs drawn in Fig 3a-b are summarised in Tables 13. Apart from the prefrontal GM region (Table 1), relaxation times were compared (see fourth columns of Tables 2 and 3) between the left and right hemispheric structures: frontal WM, parietal WM, hippocampus, and occipitoparietal region containing a GM-WM mix. For these four bilateral brain regions, the measured relaxation times were again averaged (see fifth columns of Tables 2 and 3). The uncertainties of all measurements are given in terms of the standard error (SE), calculated as a ratio of the standard deviations of the measurements to the square root of the sample size measured. The goodness of the relaxation curve fits were assessed in terms of their $R^{2}$ values generated by the nonlinear least square curve fitting routine in MATLAB; the worst $\mathrm{R}^{2}$ value was 0.74 .

All the $T_{1}$ estimates in the left hemisphere were found to be slightly greater than those in the

Table 1: Average relaxation times in the prefrontal GM region

\begin{tabular}{lll}
\hline Region $^{\text {label }}$ & $\boldsymbol{T}_{1}[\mathbf{m s}( \pm$ SE)] & $\boldsymbol{T}_{2}[\mathbf{m s}( \pm$ SE) $]$ \\
\hline Prefrontal GM $^{1}$ & $1942(29)$ & $147(9)$ \\
\hline
\end{tabular}

Table 2: $T_{1}$ relaxation times compared between left and right hemispheric ROIs drawn within four brain regions

\begin{tabular}{llcll}
\hline Region ${ }^{\text {labels }}$ & $\begin{array}{l}\text { Left } \\
\text { hemisphere } \\
{[\mathbf{m s}( \pm \text { SE) }]}\end{array}$ & $\begin{array}{l}\text { Right } \\
\text { hemisphere } \\
{[\mathbf{m s}( \pm \text { SE) }]}\end{array}$ & $\begin{array}{l}\text { Comparison } \\
(\boldsymbol{p} \text {-value })\end{array}$ & $\begin{array}{l}\text { Average } \\
{[\mathbf{m s}( \pm \text { SE) }]}\end{array}$ \\
\hline Frontal WM $^{2,3}$ & $1235(52)$ & $1170(64)$ & 0.07 & $1203(40)$ \\
Parietal WM $^{4,5}$ & $1238(21)$ & $1196(36)$ & 0.12 & $1217(21)$ \\
Occipitoparietal GM/WM $^{6,7}$ & $1441(40)$ & $1410(47)$ & 0.24 & $1425(29)$ \\
Hippocampus $^{8,9}$ & $1439(96)$ & $1432(94)$ & 0.64 & $1435(62)$ \\
\hline
\end{tabular}


Table 3: $T_{2}$ relaxation times compared between left and right hemispheric ROIs drawn within four brain regions

\begin{tabular}{lllll}
\hline Region ${ }^{\text {labels }}$ & $\begin{array}{l}\text { Left } \\
\text { hemisphere } \\
{[\mathbf{m s}( \pm \mathbf{S E})]}\end{array}$ & $\begin{array}{l}\text { Right } \\
\text { hemisphere } \\
{[\mathbf{m s}( \pm \mathbf{S E})]}\end{array}$ & $\begin{array}{l}\text { Comparison } \\
(\boldsymbol{p} \text {-value })\end{array}$ & $\begin{array}{l}\text { Average } \\
{[\mathbf{m s}( \pm \text { SE)] }}\end{array}$ \\
\hline Frontal WM $^{2,3}$ & $122(5)$ & $120(4)$ & 0.49 & $121(3)$ \\
Parietal WM $^{4,5}$ & $128(4)$ & $134(8)$ & 0.37 & $131(4)$ \\
Occipitoparietal GM/WM $^{6,7}$ & $127(1)$ & $127(2)$ & 0.88 & $127(1)$ \\
Hippocampus $^{8,9}$ & $134(15)$ & $150(5)$ & 0.27 & $142(8)$ \\
\hline
\end{tabular}

right hemisphere but this difference was not statistically significant $(p>0.05)$. The one-way ANOVA results showed that while $T_{1}$ varied significantly $(p<0.01)$ across the brain regions, regional $T_{2}$ did not vary significantly $(p=0.07)$. The prefrontal GM relaxation times were included in the respective one-way ANOVA tests.

\section{DISCUSSION}

Generally, $T_{2}$ estimates were only about a tenth of the $T_{1}$ estimates, consistent with theoretical predictions. Longest $T_{1}$ and $T_{2}$ relaxation times were observed in grey matter regions. Both $T_{1}$ and $T_{2}$ were not significantly different between the two hemispheres of the brain, indicating an approximate equality of relaxation times between contralateral sections of the brain. However, left hemispheric $T_{1}$ estimates showed some tendency of higher values than those estimated from the right hemisphere. Intrahemispheric $T_{1}$ relaxation times varied widely among the various brain structures. However, $T_{2}$ relaxation times did not vary significantly among the brain structures in both hemispheres.

At 4.0 T, Kim et al (1994) also observed that the average $T_{1}$ of GM was greater in the left than in the right hemisphere, even without performing any significance test on their results. Similarly, Wansapura et al (1999) also observed at 3.0 $\mathrm{T}$ that out of nine ROIs in each hemisphere, eight ROIs in the left had greater
$T_{1}$ estimates than the corresponding regions in the right hemisphere. They only found this difference to reach statistical significance in GM insula. Garber et al (1989) also reported higher $T_{1}$ values in the left than in the right hemispheres in GM and WM regions at $1.5 \mathrm{~T}$. These findings compare with those reported in this study.

Hemispheric $T_{2}$ differences were not significant in all brain regions considered in this study. Even though they did not study the same regions as have been studied in this report, Wansapura et al (1999) did not find any difference in $T_{2}$ between the two hemispheres for all their nine ROIs. They reported that they could not cite any literature reporting hemispheric $T_{2}$ differences. They attributed the $T_{1}$ differences they observed between the two hemispheres to non-uniformity of the $B_{1}$ field caused by the non-uniform characteristics of brain tissue. Tissue-type differences give rise to standing waves in the head which cause variation in the flip angles across the brain with the application of the RF pulses (Tofts, 2003; Helms, 2008). The extent of the standing wave effect depends on the tissue water proton density of the region of interest. Thus, Wansapura et al (1999) did not find differences in the relaxation times between left and right ROIs selected on the image of a uniform $\mathrm{MnCl}_{2}$-doped water phantom.

$T_{1}$ values in $\mathrm{GM}$ regions were observed to be 
greater than those in WM regions. Even though a similar observation has been made previously (Wansapura et al., 1999), this difference was not found to be significant in this study. The reason for this insignificant difference in GM/ WM $T_{1}$ may be due to the dependence of $T_{1}$ on $B_{0}$ field strength (Bottomley et al., 1984; Fischer et al., 1990), so that at high fields $\left(B_{0} \geq\right.$ $3.0 \mathrm{~T})$, tissue relaxation rates approach that of water resulting in low GM/WM $T_{1}$ contrast (Wansapura et al., 1999). In each hemisphere, the hippocampi, which are GM structures, showed the longest average $T_{2}$ relaxation time compared to the other structures, while the frontal WM regions had the shortest average $T_{2}$ relaxation time. This observation compares with the finding of Wansapura et al (1999); they found the highest and lowest $T_{2}$ estimates in the occipital GM and frontal WM, respectively. The shorter $T_{2}$ relaxation times in WM could be attributable to its high ferric iron content (Drayer et al., 1986), which is reported to reduce $T_{2}$ relaxation times (Drayer et al., 1986; Ye et al., 1996; Vymazal et al., 1995; Vymazal et al., 1996).

Generally, studies of hemispheric and regional differences in quantitative $T_{1}$ and $T_{2}$ measurements in the brain are not based on a consistent selection of ROIs or subjected to tests of statistical significance (Wansapura et al., 1999). It is therefore difficult to do a more rigorous comparison between the results reported here and those in the literature. Nonetheless, the results of this study are consistent with those reported in the literature for the selected brain regions at the respective field strengths.

\section{CONCLUSION}

$T_{1}$ and $T_{2}$ relaxation times have been accurately measured in nine brain regions of healthy volunteers. Generally, regional brain $T_{1}$ was observed to be slightly higher in the left hemisphere but $T_{2}$ variation did not show any clear trend between the two hemispheres. The estimates reported here are consistent with theoretical predictions and with published data for relaxation time measurements in the selected anatomical regions at various field strengths. Thus this report provides a comprehensive data set, which may prove useful as a reference for optimisation of pulse sequences for brain imaging at $3.0 \mathrm{~T}$, as well as in multicentre comparisons of measured relaxation times for the anatomical regions studied, especially for purposes of diagnostics.

\section{ACKNOWLEDGEMENT}

The authors would like to thank the Scottish Imaging Network; a Platform for Scientific Excellence (SINAPSE), University of Glasgow, and the Sackler Institute of Psychological Research, UK for jointly funding this research. The authors wish to acknowledge the dedication of the study volunteers.

\section{LIST OF ABBREVIATIONS}

ANOVA = Analysis of Variance

$\mathrm{CSF}=$ Cerebrospinal fluid

$\mathrm{GM}=$ Grey matter

$\mathrm{GE}=$ General Electric

MR/MRI = Magnetic Resonance/ Magnetic

Resonance Imaging

NMR = Nuclear magnetic resonance

$\mathrm{RF}=$ Radiofrequency

ROI = Region of interest

$T_{1}=$ Longitudinal (or spin-lattice) relaxation time

$T_{2}=$ Transverse (or spin-spin) relaxation time

$T E=$ Echo time

$T R=$ Repetition time

$\mathrm{WM}=$ White matter

\section{REFERENCES}

Bottomley, P. A., Foster, T. H., Argersinger, R. E. and Pfeifer, L. M. (1984). "A review of normal tissue hydrogen NMR relaxation times and relaxation mechanisms from 1-100 MHz: dependence on tissue type, NMR frequency, temperature, species, excision, and age". Medical Physics, 11(4): 425-48

Drayer, B., Burger, P., Darwin, R., Riederer, S., Herfkens, R. and Johnson, G. A. (1986). "MRI of brain iron". American Journal of Roentgenology, 147(1): 103-110 
Fischer, H. W., Rinck, P. A., Haverbeke, Y. V. and Muller, R. N. (1990). "Nuclear relaxation of human brain gray and white matter: analysis of field dependence and implications for MRI". Magnetic Resonance in Medicine, 16(2): $317-34$

Garber, H. J., Ananth, J. V., Chiu, L. C., Griswold, V. J. and Oldendorf, W. H. (1989). "Nuclear magnetic resonance study of obsessive-compulsive disorder". American Journal of Psychiatry 146(8): 1001-5

Helms, G. (2008). "The principles of quantification applied to in vivo proton MR spectroscopy". European Journal of Radiology, 67 (2): 218-29

Kim, S. G., Hu, X. and Ugurbil, K. (1994). "Accurate T1 determination from inversion recovery images: application to human brain at 4 tesla". Magnetic Resonance in Medicine, 31(4): 445-449

Koenig, S. H. and Brown, R. D. I. (1984). "Determinants of proton relaxation in tissues". Magnetic Resonance in Medicine, 1: 437-449

Laule, C., Vavasour, I. M., Kolind, S. H., Traboulsee, A. L., Moore, G. R. W., Li, D. K. B. and MacKay, A. L. (2007). "Long T2 water in multiple sclerosis: what else can we learn from multi-echo T2 relaxation?" Journal of Neurology, 254(11): 1579-1587

Posse, S., Cuenod, C. A., Risinger, R., Bihan, D. L. and Balaban, R. S. (1995). "Anomalous transverse relaxation in $1 \mathrm{H}$ spectroscopy in human brain at 4 tesla". Magnetic Resonance in Medicine,33: 246-252
Gowland, P. A. and Stevenson, V. L. (2003). T. I: the longitudinal relaxation time". In: Tofts, P. (Ed), "Quantitative MRI of the brain: measuring changes caused by disease". John Wiley and Sons Ltd, Chichester, England. Pp 111-141

Vymazal, J., Brooks, R. A., Baumgarner, C., Tran, V., Katz, D., Bulte, J. W. M., Bauminger, E. R. and Chiro, G.D. (1996). "The relation between brain iron and NMR relaxation times: an in vitro study". Magnetic Resonance in Medicine, 35(1): 56-61

Vymazal, J., Hajek, M., Patronas, N., Giedd, J. N., Bulte, J. W., Baumgarner, C., Tran, V. and Brooks, R. D. (1995). "The quantitative relation between T1-weighted and T2weighted MRI of normal gray-matter and iron concentration". Journal of Magnetic Resonance Imaging, 5(5): 554-560

Wansapura, J. P., Holland, S. K., Dunn, R. S. and Ball, S. W. (1999). "NMR relaxation times in the human brain at 3.0 tesla". Journal of Magnetic Resonance Imaging, 9(4): 531-538

Whittall, K. P., MacKay, A. L. and Li, D. K. (1999). "Are mono-exponential fits to a few echoes sufficient to determine T2 relaxation for in vivo human brain?" Magnetic Resonance in Medicine., 41(6): 1255-1257

Ye, F. Q., Martin, W. R. W. and Allen, P. S. (1996). "Estimation of the iron concentration in excised gray matter by means of proton relaxation measurements". Magnetic Resonance in Medicine, 35(3): 285-289 